Review article

\title{
A review on strategies to important knowledge and attitude of Iranian pregnant women requesting for caesarean
}

\author{
Nastaran Bagherian-Afrakoti ${ }^{1}$, Mehdi Pourasghar ${ }^{2}$, Marjan Ahmad shirvani ${ }^{*}$, Abbas Alipour ${ }^{4}$ \\ (Received: 15 May 2016; Accepted: 15 Aug 2016)
}

\begin{abstract}
Background and Purpose: Maternal request for cesarean section is a major influential factor in the rising trend of this childbirth mode. Lack of knowledge and negative attitudes toward natural delivery are the main causes of this issue. This study aimed to review the strategies to increase the knowledge and change the attitudes and request of Iranian pregnant women for caesarean section.

Methods: In this narrative review, all interventional studies conducted on Iranian pregnant women with the objective of enhancing the knowledge and attitudes toward natural delivery were identified via searching in databases such as Google Scholar, ScienceDirect, Scopus, SID, MedLib, Magiran, and PubMed for articles published during 2000-2015.

Results: In total, 22 articles were reviewed in this study. In these studies, systematic and planned educational interventions were carried out, including group education and discussion, question and answer and role-playing $(\mathrm{n}=10)$. Other intervention methods were based on various models and theories, such as the health belief model $(n=3)$, behavioral intention model $(n=4)$, theory of reasoned action $(n=1)$, theory of planned behavior $(n=2)$, and cognitive-behavioral consultation $(n=2)$. Reviewed findings were indicative of the significant improvement of knowledge, attitudes and intentions toward natural delivery following the interventions $(P<0.05)$. Conclusion: According to the results of this review, various education and consultation programs are effective in enhancing the knowledge and changing the attitudes and intentions of pregnant women toward natural childbirth. Nevertheless, high rate of cesarean section suggests that these interventions are not implemented consistently. It is noteworthy that effects of other confounders must be considered in this regard.
\end{abstract}

Keywords: Attitude, Caesarean section, Childbirth, Knowledge, Pregnant women

\section{Introduction}

Natural childbirth is considered the most beneficial delivery mode for most pregnant women. However, the rising trend of caesarean section has led to the decline of the rate of natural delivery (1). Exponential growth rate of caesarean section is reported in both developed and developing countries, which is a major concern among health experts, especially in developing countries $(2,3)$. Given the importance of reducing the rate of $\mathrm{C}$-section, specific supervision and effective strategies are required in this regard (4).

According to the World Health Organization (WHO), rate of caesarean section was predicted to reach $10-15 \%$ in $2012(5,6)$. In Iran, rate of C-section has soared to 3-4 times higher than the international standards $(7,8)$. As such, approximately $40 \%$ and $90 \%$ of the deliveries in governmental and private

\footnotetext{
${ }^{1}$ Student Research Committee, Nursing and Midwifery Faculty, Mazandaran University of Medical Sciences, Sari, Iran.

${ }^{2}$ Psychiatry and Behavioral Sciences Research Center, Addiction Institute, Department of Psychiatry, Mazandaran University of Medical Sciences, Sari, Iran

$3^{3,}$ Corresponding author: Departement of Midwifery, Nursing and Midwifery Faculty, Mazandaran University of Medical Sciences, Sari, Iran. Email: shirvani44@yahoo.com

${ }^{4}$ Department of Community Medicine, School of Medicine, Mazandaran University of Medical Sciences, Sari, Iran
} 
hospitals account for caesarean section, respectively $(9,10)$. Today, increased rate of caesarean section has been attributed to cases without obstetric or medical complications only on maternal request $(11,12)$.

Domestic studies have demonstrated that 11$22 \%$ of caesarean deliveries are performed on maternal request $(12,13)$. Ideally, caesarean section is considered in cases where natural delivery is not possible or poses great risk to the health of mother and infant (14). Similar to other major surgeries, $\mathrm{C}$-section without medical indications is associated with severe consequences, which might be lifethreatening in some cases (15-17).

On the other hand, international studies in this regard have shown that the majority of pregnant women have positive attitudes and adequate knowledge regarding natural childbirth. As such, natural childbirth is the preferred mode of delivery for various reasons, such as less pain after natural delivery compared to caesarean section $(18,19)$. By contrast, related studies in Iran have denoted the inadequate knowledge of pregnant women regarding natural delivery and $\mathrm{C}$-section, which stems from false beliefs about caesarean section and negative attitudes toward natural childbirth (20-23).

With this background in mind, countries with higher $\mathrm{C}$-section rate than the international standards, such as Iran, require effective strategies for the proper selection of delivery modes through improving the knowledge and attitudes of pregnant women. According to the literature, interventions focusing on the enhancement of knowledge and attitude of pregnant women toward natural delivery result in the mental and spiritual preparation of these women to select natural delivery and cope with labor. Ultimately, this leads to the reduction of unnecessary caesarean sections $(6,20,24)$.

Despite extensive research to assess the results of various interventions on maternal knowledge, attitude, and request for delivery modes, no review studies could be found on this specific subject. Available reviews in this regard only focused on the reduction of C-section rate or transforming the knowledge of pregnant women through different strategies (25-27).

For instance, Cochrane evaluated non-clinical interventions conducted to decrease unnecessary
C-sections by 2010 . Nevertheless, evidence is scarce is this regard, and further studies are required in different populations (25). Moreover, another review focusing on medical and non-medical strategies to reduce the rate of cesarean section indicated that evidence is lacking regarding the efficacy of prenatal interventions (26).

Another Cochrane review in 2007 assessed the results of three studies on the beneficial effects of educational interventions on the knowledge of pregnant women about modes of delivery. However, studies conducted on the Iranian populations have received insufficient attention in all the aforementioned reviews (27).

Considering the high rate of caesarean section in Iran, inadequate knowledge and negative attitudes toward natural delivery, key role of maternal request in increased rate of C-section, and lack of recent review studies in this regard, especially in Iran, this narrative review aimed to identify and summarize the evidence about the efficacy of various strategies in enhancing the knowledge and attitudes of Iranian pregnant women toward caesarean section.

\section{Materials and Methods}

This narrative review aimed to explore the studies focusing on the changes in the knowledge, attitude, and request of pregnant women toward delivery modes through prenatal interventions.

\section{Identification of research questions}

We assessed the published evidence in order to address the following questions:

1. Which prenatal interventions are effective in enhancing the knowledge of pregnant women toward childbirth modes?

2. Which prenatal interventions are effective in improving the attitude of pregnant women toward natural delivery?

3. Which prenatal interventions are effective in changing the request of pregnant women for cesarean section?

\section{Search strategy}

Literature review was performed via searching in 
databases such as Google Scholar, ScienceDirect, Scopus, SID, MedLib, Magiran, and PubMed using specialized keywords (knowledge, attitude, pregnant women, childbirth and caesarean section). To find additional articles, we also searched the references of the selected studies. Article search and assessment were carried by two reviewers independently. Initially, abstracts of the selected articles were reviewed in terms of the inclusion and exclusion criteria. Afterwards, relevant articles were evaluated in full text.

\section{Eligibility criteria and article selection}

Abstracts of the selected articles were reviewed by two researchers to screen for duplication and verify the eligibility of data. Eligibility criteria for selection were articles published in Persian or English and studies conducted on Iranian women during 2000-2015. After final approval through the review of full-text articles, data extraction was performed $(28,29)$.

\section{Inclusion criteria}

Inclusion criteria were interventional studies (e.g., clinical trials, field trials, experimental and quasi-experimental studies) aiming to improve the knowledge, attitude and request of pregnant women regarding the mode of delivery, relevant articles published in Persian or English, and studies conducted on Iranian women.

\section{Exclusion criteria}

Exclusion criteria of the study were as follows: 1) unoriginal and non-interventional studies (e.g., descriptive articles, case-controls, cohort studies);

2) articles not addressing the research questions;

3) studies exclusively focused on pregnant women's intentions or rate of delivery modes; 4) interventional studies conducted to enhance the knowledge and attitudes of pregnant women toward vaginal birth after $\mathrm{C}$-section (VBAC); 5) studies focused on the knowledge and attitude toward VBAC and 6) studies with postnatal interventions.

\section{Data collection}

Extracted data from the articles were organized in accordance with the review questions. Moreover, types and methods of the applied interventions were classified. In this regard, we summarized the differences in the mean and percentage of the scores of knowledge and attitude in pregnant women, as well as maternal request for cesarean section, pre- and post-intervention results, and comparisons with control subjects. In addition, type of studies, educators, and features of study subjects were outlined for this narrative review.

\section{Results}

In the literature review, 91 articles were found in various electronic databases, including ScienceDirect $(n=8)$, Scopus $(n=3)$, SID $(n=7)$, Magiran ( $n=14)$, PubMed ( $\mathrm{n}=12)$, MedLib ( $\mathrm{n}=10)$, and Google Scholar $(\mathrm{n}=37)$. After reviewing the titles and abstracts, 26 articles were excluded due to duplication, and 65 articles were assessed. After selecting 11 articles by reviewing the references, 75 articles remained in the study.

Out of the remaining articles, four cases were not available in full text, and titles and abstracts of 25 cases were not consistent with the review questions. In total, 29 articles were excluded from the study. In addition, out of 47 articles available in full text, 25 cases were irrelevant. Finally, 22 articles were evaluated in this review (Figure 1), 13 of which were published in Persian and nine cases were published in English.

Among the selected articles, three cases were clinical trials $(6,30,31)$, one study was a field trial (32), and the others were experimental or quasiexperimental studies. In these studies, educational interventions for pregnant women were implemented by graduated midwives and qualified health educators, with the exception of two studies, in which the interventions were performed by a psychologist and community health providers $(24,33)$. As for sample populations, more than $90 \%$ of the subjects were nulliparous women aged less than 35 years. In the majority of the studied women, gestational age was over 24 weeks, and none of the cases had gestational age of more than 36 weeks. It is noteworthy that only one research was conducted during the first trimester 


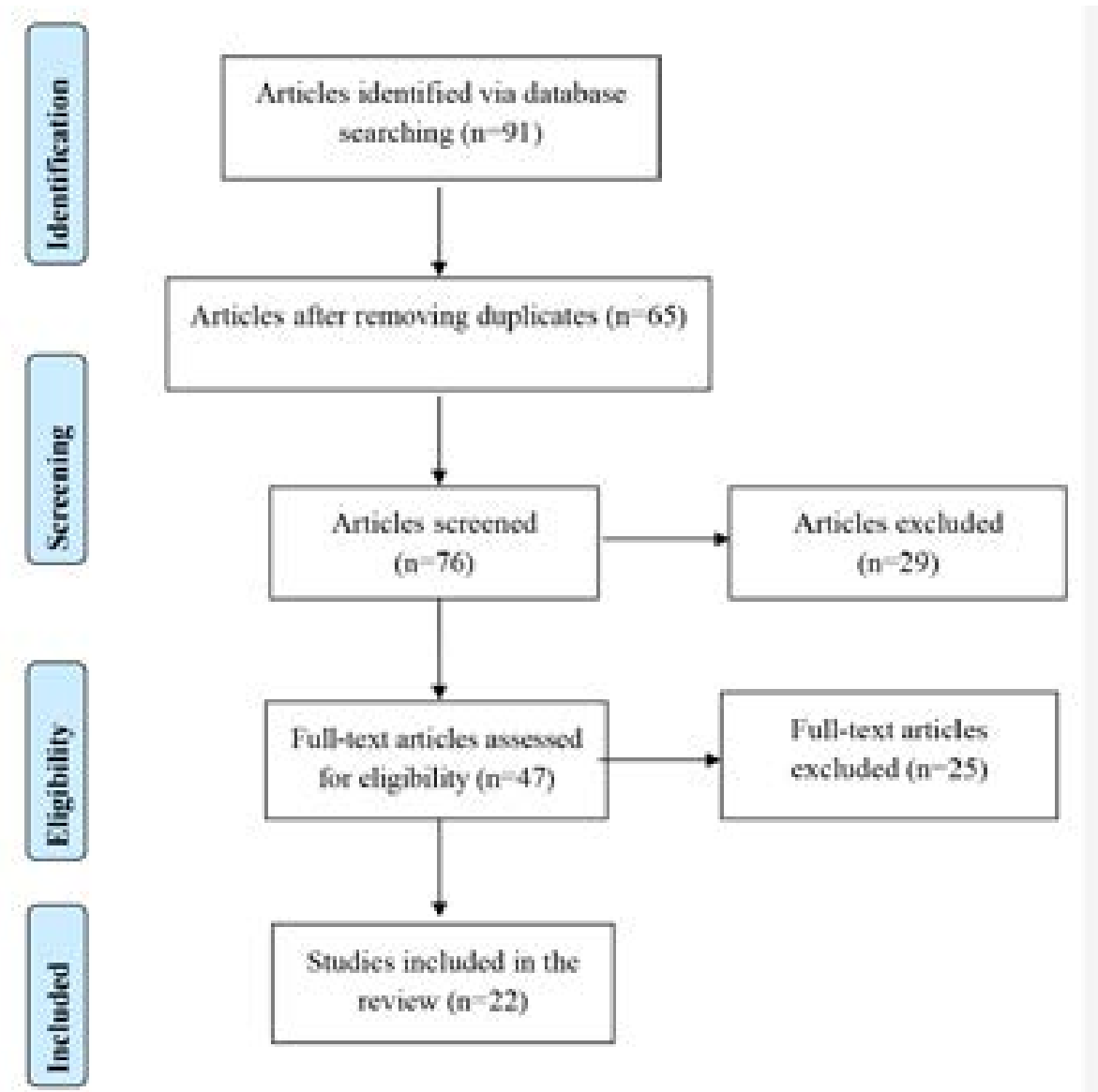

Figure 1. Process of article selection

of pregnancy (32).

Based on this review, interventions were classified into three categories. The first category included 10 articles with systematic and planned educational interventions using different methods, the second category consisted of 10 articles with interventions based on models and theories, and the third category included two articles with consultation-based interventions (Table 1). All the reviewed studies had control groups (routine care), with the exception of three cases, in two of which within-group comparison was performed before and after the interventions $(34,35)$. Moreover, in one study, two interventional groups were compared (30).

According to the results of this review, all the educational interventions (i.e., planned education and training based on models and theories) significantly enhanced the knowledge of pregnant women. However, none of the studies with consultation-based interventions reported changes in the knowledge of

Table 1. Types of interventions implemented in reviewed articles

\begin{tabular}{lc}
\hline Type of intervention & Methodology \\
\hline Systematic and planned educational intervention & Group education, group discussion, question and answer, lecture and role-playing ( $\mathrm{n}=10)$ \\
\hline & $\begin{array}{c}\text { Health belief model }(\mathrm{n}=3) \\
\text { Behavioral intention model }(\mathrm{n}=4) \\
\text { Theory of reasoned action }(\mathrm{n}=1) \\
\text { Theory of planned behavior }(\mathrm{n}=2)\end{array}$ \\
\hline Educational intervention based on models and theories & Cognitive-behavioral therapy (n=2) \\
\hline
\end{tabular}


the subjects.

Among the reviewed studies, two cases compared the effects of two different interventions on the level of knowledge. In one of these studies, educational booklets alone were compared with combined training through group discussion and booklets. According to the results, the second training method was more effective in improving the knowledge of pregnant women (36). In another research, lectures were reported to increase knowledge more significantly compared to role-playing (30).

According to the results of this narrative review, all the applied interventions (systematic education, education based on models, theories and consultation) caused a significant improvement in the attitude of pregnant women toward delivery modes. On the other hand, positive effects of the health belief model and theory of reasoned action on attitude were reported by none of the reviewed studies.

Educational intervention through the combination of booklets and group discussion was found to be more effective in improving attitudes compared to educational booklets alone (36). Furthermore, effect of lectures was reported to be more remarkable compared to role-playing in enhancing the attitude of pregnant women toward delivery modes (30).

Among the selected articles, seven studies assessed the rate of $\mathrm{C}$-section after educational interventions. Correspondingly, interventions based on systematic education $(6,35,37,38)$, theory of planned behavior (20), behavioral intention model (39), and health belief model (40) decreased the rate of C-section. Other studies reported that intentions for natural delivery increased following educational interventions or consultation. Furthermore, group consultation along with booklets was reported to be more effective in encouraging natural delivery compared to booklets alone (36). Role-playing was found to be more effective in increasing intentions for natural childbirth compared to lectures (30) (Table 2).

Table 2. Summarized results of interventional studies on enhancing knowledge, attitude and, request for C-section in Iranian pregnant women

\begin{tabular}{|c|c|c|c|c|}
\hline $\begin{array}{l}\text { Author } \\
\text { (year) }\end{array}$ & Participants/sample size & Study design & Type of intervention & Most important results \\
\hline $\begin{array}{l}\text { Lashgari } \\
(2005)(31)\end{array}$ & $\begin{array}{l}200 \text { nulliparous women with } \\
\text { gestational age of }<30 \text { weeks } \\
\text { (intervention }=100 \\
\text { control }=100 \text { ) }\end{array}$ & $\begin{array}{l}\text { Single-blind } \\
\text { randomized } \\
\text { clinical trial }\end{array}$ & $\begin{array}{l}6 \text { educational sessions until week } 36 \\
\text { of gestation using films and pamphlets } \\
\text { (control group: routine care; follow-up: } \\
36^{\text {th }} \text { week) }\end{array}$ & $\begin{array}{c}\text { Education group vs. control group: } \\
\text { knowledge about natural delivery: } 75 \% \text { vs. } 4 \% / \mathrm{C} \text {-section: } \\
71 \% \text { vs. } 9 \%(P<0.0001) \\
\text { Changes in intention for C-section: } 39 \% \text { to } 25 \% \text { vs. } 40 \% \\
\text { to } 41 \%(P=0.02)\end{array}$ \\
\hline $\begin{array}{l}\text { Fathian } \\
(2007)(41)\end{array}$ & $\begin{array}{l}140 \text { nulliparous women in } \\
\text { third pregnancy trimester } \\
\text { (intervention=70, } \\
\text { control }=70 \text { ) }\end{array}$ & Experimental & $\begin{array}{l}6 \text { educational sessions based on model } \\
\text { of behavioral intention with lectures } \\
\text { and group discussion } \\
\text { (control group: routine prenatal care; } \\
\text { follow-up: after } 3 \text { months) }\end{array}$ & $\begin{array}{l}\text { Score changes of education group vs. control group: } \\
\text { knowledge: } 56.8 \pm 20.6 \text { to } 87.1 \pm 14.5 \text { vs. } 48.7 \pm 18.5 \text { to } 50 \pm 23 \\
(P<0.001) / \text { attitude: } 55.1 \pm 12.8 \text { to } 66.5 \pm 10.7 \text { vs. } 56.8 \pm 13.3 \text { to } \\
52.5 \pm 16.2(P=0.004) / \text { intention for natural delivery: } 74.3 \% \\
\text { to } 92.9 \% \text { vs. } 71.4 \% \text { to } 49.9 \%(P=0.007)\end{array}$ \\
\hline $\begin{array}{l}\text { Toghiani } \\
(2008)(32)\end{array}$ & $\begin{array}{l}60 \text { pregnant women with } \\
\text { gestational age of } 6-8 \text { weeks } \\
\text { (intervention }=30 \\
\text { control }=30 \text { ) }\end{array}$ & Field trial & $\begin{array}{l}14 \text { sessions of group education based } \\
\text { on requirements of different pregnancy } \\
\text { trimesters through group discussion and } \\
\text { question and answer (control group: } \\
\text { routine prenatal education; follow-up: } \\
\text { end of each trimester) }\end{array}$ & $\begin{array}{l}\text { Score changes of education group vs. control group: } \\
\text { knowledge: } 51.7 \pm 18.6 \text { to } 81.2 \pm 9.1 \text { vs. } 42.9 \pm 0.8 \text { to } 71.5 \pm 11 \\
(P=0.02) / \text { attitude: } 74.1 \pm 7.1 \text { to } 76.6 \pm 7.6 \text { vs. } 66.5 \pm 17.7 \text { to } \\
71.5 \pm 11.4(P=0.02) / \text { selecting natural delivery: } 54.1 \pm 15.3 \text { to } \\
67.1 \pm 11.1 \text { vs. } 51.8 \pm 16.1 \text { to } 55.9 \pm 12.9(P=0.006)\end{array}$ \\
\hline $\begin{array}{l}\text { Sharifirad } \\
(2009)(39)\end{array}$ & $\begin{array}{l}140 \text { nulliparous women } \\
\text { (intervention }=70 \\
\text { control }=70 \text { ) }\end{array}$ & $\begin{array}{c}\text { Quasi- } \\
\text { experimental }\end{array}$ & $\begin{array}{l}3 \text { sessions of lecture and group } \\
\text { education based on behavioral } \\
\text { intention model (in addition to one } \\
\text { session for spouses of pregnant } \\
\text { women)(control group not explained) }\end{array}$ & $\begin{array}{l}\text { Score changes of education group vs. control group: } \\
\text { knowledge: } 5.7 \pm 2.1 \text { to } 8.7 \pm 1.45 \text { vs. } 4.9 \pm 1.85 \text { to } 5 \pm 2.3 \\
(P<0.001) / \text { attitude: } 55.1 \pm 12.8 \text { to } 66.5 \pm 10.8 \text { vs. } 56.8 \pm 13.3 \text { to } \\
52.5 \pm 16.2(P=0.004) / \text { definite selection of natural delivery: } \\
25.7 \% \text { to } 68.6 \% \text { vs. } 30 \% \text { to } 37.1 \%(P=0.05) / \text { rate of natural } \\
\text { delivery: } 63.33 \% \text { vs. } 36.7 \%(P=0.02)\end{array}$ \\
\hline $\begin{array}{l}\text { Tofighi } \\
(2010)(35)\end{array}$ & $\begin{array}{c}60 \text { nulliparous women with } \\
\text { gestational age of up to } 26 \\
\text { weeks } \\
\text { (no control group) }\end{array}$ & $\begin{array}{c}\text { Quasi- } \\
\text { experimental }\end{array}$ & $\begin{array}{l}\text { 45-minute group education every } \\
10-15 \text { days according to protocol } \\
\text { of Iranian Ministry of Health and } \\
\text { Medical Education }\end{array}$ & $\begin{array}{c}\text { Before and after intervention: knowledge: } 4.44 \pm 0.76 \text { and } \\
6.56 \pm 1.58(P<0.001) / \text { attitude: } 3.34 \pm 0.35 \text { and } 3.43 \pm 0.32 \\
(P<0.001) / \text { intention for natural delivery: } 61.8 \% \text { to } 80 \% / \\
\text { overall rate of natural delivery: } 57.6 \%\end{array}$ \\
\hline $\begin{array}{l}\text { Besharati } \\
(2011)(42)\end{array}$ & $\begin{array}{l}72 \text { pregnant women with } \\
\text { gestational age of } 28-32 \\
\text { weeks } \\
\text { (intervention }=36 \\
\text { control=36) }\end{array}$ & $\begin{array}{c}\text { Quasi- } \\
\text { experimental }\end{array}$ & $\begin{array}{l}4 \text { sessions of exclusive education } \\
\text { based on theory of planned behavior } \\
\text { (50-60 minutes) using educational } \\
\text { booklets (control group: routine } \\
\text { education; follow-up: weeks } 38-40 \text { ) }\end{array}$ & $\begin{array}{c}\text { Score changes of education group vs. control group: } \\
\text { knowledge: } 47.5 \pm 12.9 \text { to } 89.2 \pm 15.19 \text { vs. } 45.5 \pm 15.85 \\
\text { to } 47.2 \pm 15.97(P=0.0001) / \text { attitude: } 66.45 \pm 12.65 \\
\text { to } 83.22 \pm 13.52 \text { vs. } 70.41 \pm 20.08 \text { to } 69.37 \pm 17.36 \\
(P=0.0001) / \text { intention for natural delivery: } 52.8 \% \text { to } \\
83.3 \% \text { vs. } 50 \% \text { to } 52.8 \%(P=0.04)\end{array}$ \\
\hline
\end{tabular}


continuous of table 2 .

\begin{tabular}{|c|c|c|c|c|}
\hline $\begin{array}{l}\text { Ghafari } \\
\text { (2011) (40) }\end{array}$ & $\begin{array}{l}100 \text { nulliparous women with } \\
\text { gestational age of } 20-30 \\
\text { weeks } \\
\text { (intervention=50, } \\
\text { control=50) }\end{array}$ & $\begin{array}{c}\text { Quasi- } \\
\text { experimental }\end{array}$ & $\begin{array}{l}6 \text { sessions of education based on } \\
\text { health belief model ( } 30-60 \text { minutes) } \\
\text { (control group: routine care; } \\
\text { follow-up: after } 4-8 \text { weeks) }\end{array}$ & $\begin{array}{c}\text { Score changes of education group vs. control group: } \\
\text { knowledge: } 54.17 \pm 16.18 \text { to } 83 \pm 6 \text { vs. } 53.97 \pm 15.46 \text { to } \\
62 \pm 14.9(P<0.001) / \text { selection and rate of delivery modes } \\
\text { not significantly different between groups }\end{array}$ \\
\hline
\end{tabular}

\begin{tabular}{|c|c|c|c|}
\hline $\begin{array}{l}\text { Abedian } \\
(2012)(30)\end{array}$ & $\begin{array}{l}67 \text { nulligravid women with } \\
\text { gestational age of } 34-36 \\
\text { weeks } \\
\text { (role-playing }=35, \text { lecture }=32 \text {, } \\
\text { control }=0 \text { ) }\end{array}$ & $\begin{array}{l}\text { Single-blind } \\
\text { randomized } \\
\text { clinical trial }\end{array}$ & $\begin{array}{c}\text { Role-playing group: } 3 \text { role-plays } \\
\text { with seven steps in one session ( } 90 \\
\text { minutes)/ lecture group: } \\
\text { education in one session ( } 90 \text { minutes) } \\
\text { (follow-up: after } 2 \text { weeks) }\end{array}$ \\
\hline
\end{tabular}

Score changes of role-playing group vs. lecture group: knowledge: $9.0 \pm 3.0$ to $17.1 \pm 4.0$ vs. $8.3 \pm 4.0$ to $18.3 \pm 5.3$ (within groups: $P=0.0001$; between groups: $P>0.05)(120 \%$ vs. $90 \%$ increase; $P=0.001$ )/ attitude: $85.4 \pm 10.1$ to $95.4 \pm 8.2$ $\begin{array}{ll}\text { (follow-up: after } 2 \text { weeks) } & \text { groups: } P=0.03 \text { )/ request for natural delivery: } 62.9 \% \text { vs. }\end{array}$ $43.8 \%(P>0.05)$

\begin{tabular}{|c|c|c|c|}
\hline $\begin{array}{l}\text { Asfia (2012) } \\
\text { (34) }\end{array}$ & $\begin{array}{l}64 \text { pregnant women } \\
\text { (intervention=64, } \\
\text { without control group) }\end{array}$ & $\begin{array}{l}\text { Interventional } \\
\text { (before-after } \\
\text { design) }\end{array}$ & $\begin{array}{l}\text { Lecture (60 minutes) with one } \\
\text { educational pamphlet } \\
\text { (follow-up: after } 2 \text { weeks) }\end{array}$ \\
\hline $\begin{array}{l}\text { Kashefi } \\
(2013)(43)\end{array}$ & $\begin{array}{l}100 \text { nulliparous women in } \\
\text { third pregnancy trimester } \\
\quad \text { (intervention }=50, \\
\text { control }=50)\end{array}$ & $\begin{array}{c}\text { Quasi- } \\
\text { experimental }\end{array}$ & $\begin{array}{l}3 \text { sessions of education based on } \\
\text { behavioral intention model } \\
\text { (control group: routine prenatal care; } \\
\text { follow-up: after } 3 \text { months) }\end{array}$ \\
\hline
\end{tabular}

\begin{tabular}{|c|c|c|c|}
\hline $\begin{array}{l}\text { Rezakhani } \\
\text { (2013) (33) }\end{array}$ & $\begin{array}{l}140 \text { nulliparous women with } \\
\text { gestational age of } 28 \text { weeks } \\
\text { (intervention }=70, \\
\text { control }=70 \text { ) }\end{array}$ & Experimental & $\begin{array}{l}2 \text { sessions of education based on } \\
\text { behavioral intention model with } \\
\text { pamphlets (control group: routine } \\
\text { prenatal care; } \\
\text { follow-up: after one month) }\end{array}$ \\
\hline
\end{tabular}

Pre- and post-intervention scores:
knowledge: $46.6 \pm 5.7$ and $54.1 \pm 5.8(P<0.001) /$
attitude: $47.6 \pm 7.04$ and $49.54 \pm 5.8(P>0.05)$

Score changes of education group vs. control group: knowledge: $54.2 \pm 19.4$ to $91.1 \pm 13.7$ vs. $55.7 \pm 19.7$ to $56.3 \pm 22.1(P<0.001) /$ attitude: $54.9 \pm 12.7$ to $74.1 \pm 9.6$ vs. $55.2 \pm 12.8$ to $52.5 \pm 16.2(P=0.003) /$ intention for natural delivery: $74 \%$ to 96 vs. $76 \%$ to $52 \%(P=0.006)$

Score changes of education group vs. control group: knowledge (median): 10 to 11 vs. 8.5 to $9(P<0.001)$ attitude (median): 23 to 27 vs. 24 to $21(P<0.001) /$ intention for natural delivery: $45.7 \%$ to $38.6 \%$ vs. $57.1 \%$ follow-up: after one month) to $37.1 \%(P>0.05)$
(

Shahraki-
$\begin{aligned} & \text { Sanavi } \\ & \text { (2014) (20) }\end{aligned}$
$\begin{gathered}\text { pregnant women in third } \\ \text { (intervention }=100, \\ \text { control=100) }\end{gathered}$

One session of group education based on theory of planned behavior Quasi- $\quad$ (60 minutes) with an educational experimental package (control group: routine prenatal care;

follow-up: after one month)
Score changes of education group vs. control group: knowledge: $9.90 \pm 2.54$ to $12.30 \pm 1.92$ vs. $10.6 \pm 2.81$ to $10.68 \pm 2.54(P<0.001) /$ attitude: $21.98 \pm 4.39$ to $24.78 \pm 5.38$ vs. $22.90 \pm 3.73$ to $22.57 \pm 4.17(P<0.001) /$ selecting natural delivery: $26 \%$ vs. $0 \% /$ natural delivery rate: $22 \%$ vs. $4 \%(P<0.001)$

\begin{tabular}{|c|c|c|c|c|}
\hline $\begin{array}{l}\text { Jeihooni } \\
(2014)(44)\end{array}$ & $\begin{array}{l}100 \text { nulliparous women in } \\
\text { third pregnancy trimester } \\
\quad \text { (intervention }=50 \\
\text { control }=50)\end{array}$ & $\begin{array}{c}\text { Quasi- } \\
\text { experimental }\end{array}$ & $\begin{array}{l}3 \text { sessions of education based on } \\
\text { theory of reasoned action } \\
\text { (control group: routine care; } \\
\text { follow-up: after } 3 \text { months) }\end{array}$ & $\begin{array}{l}\text { Score changes of education group vs. control group: } \\
\text { knowledge: } 5.41 \pm 1.9 \text { to } 9.11 \pm 1.3 \text { vs. } 5.57 \pm 1.9 \text { to } \\
5.63 \pm 2.2(P=0.001) / \text { intention for natural delivery: } 74 \% \\
\text { to } 96 \% \text { vs. } 76 \% \text { to } 52 \%(P=0.001)\end{array}$ \\
\hline $\begin{array}{l}\text { Shahraki- } \\
\text { Sanavi } \\
(2014)(36)\end{array}$ & $\begin{array}{l}200 \text { pregnant women in } \\
\text { third pregnancy trimester } \\
\text { (educational package } \\
\text { group }=40, \\
\text { educational package and } \\
\text { group discussion }=60, \\
\text { control }=100 \text { ) }\end{array}$ & $\begin{array}{c}\text { Quasi- } \\
\text { experimental }\end{array}$ & $\begin{array}{l}\text { One group received an educational } \\
\text { package (three booklets) and the } \\
\text { other group received an educational } \\
\text { package and group discussion ( } 45 \\
\text { minutes) (control group: routine care; } \\
\text { follow-up: after one month) }\end{array}$ & $\begin{array}{c}\text { Score changes of educational package group vs. discussion } \\
\text { and control groups: knowledge: } 10.35 \pm 2.63 \text { to } 12.38 \pm 1.87 \\
\text { vs. } 9.78 \pm 2.53 \text { to } 12.40 \pm 1.87 \text { and } 9.95 \pm 2.70 \text { to } 10.59 \pm 2.52 \text {, } \\
\text { respectively }(P<0.001) / \text { attitude: } 22.45 \pm 4.30 \text { to } 23.83 \pm 4.45 \\
\text { vs. } 22.03 \pm 4.23 \text { to } 25.80 \pm 5.63 \text { and } 22.68 \pm 3.94 \text { to } 22.34 \pm 4.20 \\
\text { respectively }(P<0.001) / \text { intention for natural delivery: } 2.5 \% \\
\text { vs. } 41.7 \% \text { and } 0 \% \text {, respectively }\end{array}$ \\
\hline $\begin{array}{l}\text { Safari } \\
\text { Moradabadi } \\
(2014)(38)\end{array}$ & $\begin{array}{c}70 \text { nulliparous women with } \\
\text { gestational age of } 25-30 \\
\text { weeks } \\
\text { (intervention }=35 \\
\text { control }=35 \text { ) }\end{array}$ & Interventional & $\begin{array}{l}\text { Lecture, group discussion, and question } \\
\text { and answer in several sessions (50- } \\
60 \text { minutes) (control group: routine } \\
\text { prenatal education; follow-up: one } \\
\text { week before delivery) }\end{array}$ & $\begin{array}{l}\text { Score changes of education group vs. control group: } \\
\text { knowledge: } 40 \pm 13.77 \text { to } 71.75 \pm 13.10 \text { vs. } 39.85 \pm 17.68 \text { to } \\
42.14 \pm 14.71(P<0.001) / \text { intention for natural delivery: } 45 \% \\
\text { vs. } 17.3 \% / \text { rate of natural delivery: } 31.42 \% \text { vs. } 11.42 \%\end{array}$ \\
\hline $\begin{array}{l}\text { Malakooti } \\
(2014)(5)\end{array}$ & $\begin{array}{c}200 \text { nulliparous women with } \\
\text { gestational age of } 24-32 \\
\text { weeks } \\
\text { (intervention=100, } \\
\text { control=100) }\end{array}$ & $\begin{array}{c}\text { Quasi- } \\
\text { experimental }\end{array}$ & $\begin{array}{l}4 \text { weekly sessions of group education } \\
\text { (control group: routine care; } \\
\text { follow-up: during last session) }\end{array}$ & $\begin{array}{c}\text { Score changes of education group vs. control group: } \\
\text { knowledge: } 2.8 \pm 3.9 \text { to } 12.3 \pm 4.6 \text { vs. } 3.6 \pm 4.8 \text { to } 4.1 \pm 4.7 \\
(P<0.001) / \text { attitude: } 47.24 \pm 8.5 \text { to } 55.0 \pm 6.0 \text { vs. } 46 \pm 10.5 \text { to } \\
47.0 \pm 10.0(P<0.001)\end{array}$ \\
\hline $\begin{array}{l}\text { Baghiani } \\
\text { Moghaddam } \\
(2014)(45)\end{array}$ & $\begin{array}{l}130 \text { nulligravid women with } \\
\text { gestational age of }>28 \text { weeks } \\
\text { (intervention }=65 \\
\text { control }=65 \text { ) }\end{array}$ & Experimental & $\begin{array}{l}5 \text { sessions of education based on } \\
\text { health belief model ( } 45 \text { minutes) } \\
\text { (control group: routine care; } \\
\text { follow-up: after } 2 \text { months) }\end{array}$ & $\begin{array}{l}\text { Score changes of education group vs. control group: } \\
\text { knowledge: } 6.14 \pm 3.65 \text { to } 11.09 \pm 3.20 \text { vs. } 3.57 \pm 3.10 \text { to } \\
4.67 \pm 2.68(P=0.001) / \text { intention for natural delivery: } \\
83.1 \% \text { vs. } 78.5 \%(P>0.05)\end{array}$ \\
\hline $\begin{array}{l}\text { Valiani } \\
\text { (2014) (6) }\end{array}$ & $\begin{array}{l}90 \text { nulliparous women }(180 \\
\text { couples) with gestational age } \\
\text { of } 26-32 \text { weeks } \\
\text { (group one }=60 \text { women, } \\
\text { group two }=60 \text { couples, } \\
\text { control }=60 \text { ) }\end{array}$ & Clinical trial & $\begin{array}{l}\text { Educational workshop in } 3 \text { weeks } \\
\text { (4-hour sessions) with lectures, role- } \\
\text { playing, problem-solving, question } \\
\& \text { answer, and pamphlets for both } \\
\text { intervention groups (follow-up: during } \\
\text { last session, after one month, and } 6 \\
\text { weeks after delivery) }\end{array}$ & $\begin{array}{c}\text { Scores of mothers vs. couples and control group in four } \\
\text { follow-ups: knowledge: } 13.10,21.6,21.97,21.40 \mathrm{vs.} \\
13.63,22.20,22.30,21.50 \text {, and } 13.27,14.47,15.10 \text {, and } \\
15.03(P<0.0001) / \text { attitude: } 47.23,76.20,85.7,86.13 \\
\text { vs. } 48.70,92.80,97.73,98.07 \text {, and } 46.67,51.6,53.8, \\
\text { and } 52.2(P<0.0001) / \text { rate of natural delivery: } 60 \% \text { vs. } \\
56.7 \% \text {, and } 26.7 \%(P=0.01)\end{array}$ \\
\hline
\end{tabular}


continuous of table 2 .

\begin{tabular}{|c|c|c|c|c|}
\hline $\begin{array}{l}\text { Abedi } \\
(2015)(46)\end{array}$ & $\begin{array}{l}24 \text { pregnant women with } \\
\text { gestational age of } 24-30 \\
\text { weeks (intervention=12, } \\
\text { control=12) }\end{array}$ & $\begin{array}{c}\text { Quasi- } \\
\text { experimental }\end{array}$ & $\begin{array}{l}8 \text { weekly sessions of cognitive- } \\
\text { behavioral consultation ( } 2 \text { hours) } \\
\text { (control group: routine care) }\end{array}$ & $\begin{array}{c}\text { Score changes of education group vs. control group: } \\
\text { attitude: } 108.33 \pm 8.62 \text { to } 113.5 \pm 6.62 \text { vs. } 103.08 \pm 6.86 \text { to } \\
103.08 \pm 5.45(P<0.05) / \text { intention for natural delivery: } \\
100 \% \text { vs. } 25 \%\end{array}$ \\
\hline $\begin{array}{l}\text { Shahnazi } \\
(2015)(47)\end{array}$ & $\begin{array}{l}88 \text { nulliparous women in } \\
\text { third pregnancy trimester } \\
\quad \text { (intervention }=44, \\
\text { control }=44 \text { ) }\end{array}$ & Experimental & $\begin{array}{l}3 \text { sessions of lecture and group } \\
\text { discussion based on health belief model } \\
\text { (one hour) (control group: routine care; } \\
\text { follow-up: after 4-8 weeks) }\end{array}$ & $\begin{array}{l}\text { Score changes of education group vs. control group: } \\
\text { knowledge: } 32.1 \pm 30.5 \text { to } 89.1 \pm 20.1 \text { (after } 4 \text { weeks) and } \\
93.6 \pm 12.6 \text { (after } 8 \text { weeks) vs. } 17.8 \pm 17.6 \text { to } 30.5 \pm 17.9 \\
\text { (after } 4 \text { weeks) and } 31.5 \pm 2.68 \text { (after } 8 \text { weeks) }(P<0.001)\end{array}$ \\
\hline $\begin{array}{l}\text { Amini } \\
(2015)(24)\end{array}$ & $\begin{array}{l}40 \text { nulliparous women with } \\
\text { gestational age of }>12 \text { weeks } \\
\text { (intervention }=20 \\
\text { control }=20 \text { ) }\end{array}$ & $\begin{array}{c}\text { Quasi- } \\
\text { experimental }\end{array}$ & $\begin{array}{l}8 \text { sessions of cognitive-behavioral } \\
\text { consultation ( } 90 \text { minutes) } \\
\text { (control group: routine care) }\end{array}$ & $\begin{array}{l}\text { Score changes of consultation group vs. control group: } \\
\text { attitude: } 89.20 \pm 6.01 \text { to } 102.95 \pm 5.19 \text { vs. } 89.35 \pm 5.36 \text { to } \\
90.55 \pm 5.59(P<0.001) / \text { intention for natural delivery: } 5 \text { to } \\
16 \text { vs. } 7 \text { to } 8(P<0.008)\end{array}$ \\
\hline $\begin{array}{l}\text { Arefi (2015) } \\
(37)\end{array}$ & $\begin{array}{l}140 \text { nulliparous women in } \\
\text { third pregnancy trimester } \\
\text { (intervention }=70, \text { control }=70 \text { ) }\end{array}$ & $\begin{array}{c}\text { Quasi- } \\
\text { experimental }\end{array}$ & $\begin{array}{l}\text { Educational intervention based } \\
\text { on *BASNEF (control group not } \\
\text { explained) }\end{array}$ & $\begin{array}{l}\text { Score changes in education group vs. control group: } \\
\text { knowledge: } 43.5 \pm 6.2 \text { to } 82.5 \pm 10(P<0.001) \text { vs. } 42.1 \pm 11 \\
\text { to } 45 \pm 15.7(P>0.05) / \text { attitude: } 55.5 \pm 12.2 \text { to } 66.5 \pm 11.1 \\
(P<0.001) \text { vs. } 54.4 \pm 13.1 \text { to } 52.1 \pm 15.9(P>0.05) / \\
\text { rate of natural delivery: } 74.3 \% \text { vs. } 40 \%(P<0.001)\end{array}$ \\
\hline
\end{tabular}

*BASNEF: Belief, attitude, subjective norm, and enabling factors

\section{Discussion}

According to the results of the present review, various educational methods have been applied to change the attitude, knowledge and request of Iranian women regarding delivery modes; among these approaches, consultation has been employed less frequently. On the other hand, few interventional studies have been conducted in this regard in other countries, most of which have assessed the changes in the intention and practice of pregnant women for $\mathrm{C}$-section or their knowledge and attitude toward $\operatorname{VBAC}(25,27)$.

This could be due to the differences in the common causes of elective $\mathrm{C}$-section across the world. While in developed communities researchers have often focused on reducing the medical causes of C-section, other studies have mostly aimed to change knowledge and attitudes in this regard. This is because in certain countries, antenatal preparation is associated with lack of structure and formality, and healthcare providers are not considered the primary sources of information for pregnant women (27).

Cultural values, beliefs, behaviors, and misconceptions play a major role in maternal request for C-section (46). As such, improvement of knowledge and attitude is of paramount importance in populations where the rate of maternal request for $\mathrm{C}$-section is relatively high.

\section{Effect of educational interventions on the} knowledge of women regarding delivery modes

Systematic education based on models and theories has proven effective in enhancing the knowledge of women regarding the modes of delivery. Moreover, increased knowledge has been reported in control groups (prenatal routine care), which could be attributed to the non-systematic training of mothers through receiving practical care, as well as the willingness of subjects for obtaining further information about related questionnaires. Therefore, education must be prioritized in the provision of prenatal and maternal care (9).

Antenatal education has multiple goals, such as the promotion of health behaviors (27). In studies conducted on various populations, educational interventions have been shown to raise the level of knowledge remarkably (27, 48-50). However, according to the Cochrane review, quality of the published articles in this area is unsatisfactory, so that the exact effects of educational interventions remain unclear (27).

The present review was similar to the experiments conducted in other regions of the world, such as North America and Europe, in which psychoprophylaxis was the foundation of most educational interventions to increase the rate of natural birth in Iran (27). As some studies have implemented paternal education (27), findings of an article in this regard 
demonstrated the enhanced knowledge of fathers as a result of couple education compared to maternal training alone. Therefore, it could be concluded that prenatal educational programs should be performed on fathers as well.

According to the current review, lecture is the most common educational method applied in previous studies to promote knowledge even though it is associated with passive learning (30). Passive educational methods (e.g., lecture) improve the level of knowledge immediately, while this improvement may not be sustained in the long run (30). In one study, reduction of knowledge was observed after a long-term follow-up in the postpartum period despite initial rising after education during pregnancy (6). Although role-playing mostly relies on the active participation of learners, results of another study indicated that enhancement of knowledge was more significant through lectures compared to this approach (30).

In studies based on planned educational interventions using various instruments (e.g., group discussion, displaying films, pamphlets, PowerPoint presentations), maternal knowledge has been reported to increase remarkably $(5,6,31,32,34,36,38)$; correspondingly, application of combined educational methods could be more effective in this regard. Findings of a research suggest that group discussion with educational packages could raise the awareness of mothers more significantly than using educational packages alone (36). Nevertheless, evidence is scarce regarding the exact effect of prenatal education, booklets and group discussion on the level of maternal knowledge (25).

Theories are essentially involved in the process of education and training. According to the present review, maternal knowledge significantly increases with the educational interventions that are based on the behavioral intention model, as well as the theory of reasoned action, which is the groundwork of the behavioral intention model $(33,39,41,43$, 44). Furthermore, the health belief model has been reported to raise the awareness of mothers toward the modes of delivery $(40,45,47)$.

These results indicate that although behavioral models are mainly applied to change attitudes and behaviors, they are effective in the enhancement of knowledge as well. According to the findings of two studies, maternal education based on the theory of planned behavior significantly improved the knowledge of pregnant women regarding childbirth modes $(20,42)$. It is noteworthy that the extent of improved knowledge has been reported variably in different studies, so that this increase was lower in one of these studies (with one training session) compared to another one. Therefore, implementation of long-term educational interventions could be associated with higher level of maternal knowledge regarding the modes of delivery.

\section{Effect of educational interventions on the attitude of women toward delivery modes}

According to the results of our review, attitude of pregnant women toward natural delivery improves after educational interventions involving systematic training programs, models and consultation. Since human attitude is based on knowledge, educational programs could be largely influential in the enhancement of knowledge, as observed in the current review (51).

In this regard, only one study used group discussion in each trimester of pregnancy, demonstrating no significant improvement in the attitudes of the women in the intervention group (32). However, the mentioned research was low-quality, and the increase in knowledge was not significant compared to the control group.

Couple education and combination of group discussion with educational packages have been shown to remarkably improve attitudes toward delivery modes $(6,36)$. However, in studies employing traditional educational methods, scores of attitude have been reported to drop or remain unchanged (39). It seems that change of attitude through the active participation of learners could be sustained in the long run (30). However, since the reviewed studies had no long-term follow-up, this finding remains disputable.

\section{Effect of educational interventions on the intention of women for natural delivery and rate of $C$-section}

According to the current review, educational and consultation-based interventions could 
increase the rate of maternal request for natural delivery; however, the difference with the control group was not significant in some studies $(33,40)$. Results of another review suggested that evidence regarding the effects of non-clinical interventions on the rate of unnecessary C-section was only available in studies with small sample sizes, and such interventions must be performed on various populations (25)

Findings in this regard could be influenced by different factors, including the teaching skills of educators, educational contents, adequacy of programs, and range of changes in knowledge and attitude. Behavior is a multifactorial phenomenon, requiring special attention in different aspects (37). According to the literature, promoting knowledge and attitude through systematic education could lead to the alteration of health behaviors (32).

Education affects behavior when it changes the attitude (35). Attitude affects the behaviors and actions of individuals since it is an intermediary between the received information and individual's response to information (30). Moreover, intentions of individuals for a certain action are resulted from both their attitudes and abstract norms (i.e., individual and social factors) (41).

Results of the reviewed studies indicated that systemic education could increase the intention of pregnant women for natural delivery. Such interventions are effective through raising maternal awareness regarding their potential abilities for natural childbirth. These findings have been confirmed by various researchers across the world $(48,52)$.

Role-playing helps learners in realizing their social role and proper decision-making under complex circumstances (53). This method has been reported to be more effective compared to lecture in the selection of natural birth by pregnant women (30). In contrast with traditional approaches, roleplaying emphasizes on changing the attitude before changing behaviors, initially focusing on the alteration of behaviors. This encourages individuals to adjust their attitude with their new behaviors (30).

According to our review, combination of group discussion and educational packages is more effective in increasing maternal intention for natural delivery compared to the use of educational booklets alone (36). Furthermore, results of another review confirmed the efficacy of this approach in reinforcing the effects of each of these methods separately (27).

The health belief model focuses on changing personal beliefs, emphasizing that the individuals are motivated by their perceptions, thereby adopting different behavioral tendencies $(40,45,47,54)$. According to the behavioral intention model, decision-making mainly relies on the logical review of information and taking the consequences of an action into account $(33,41,43)$. Theory of planned behavior is based on behavioral patterns, attitudes toward behaviors, abstract norms, and control of perceived behaviors $(55,56)$. With this background in mind, it could be inferred that theory-based educational interventions could affect intentions through transforming attitudes and behaviors.

Behavioral-cognitive consultation helps individuals to recognize their misconceptions and succeed in proper decision-making $(24,46,57,58)$. Review of theliterature revealed that consultation is significantly effective in altering maternal intention for natural delivery, as in a study, all the participants of the consultation group opted for natural delivery. In addition, rate of natural delivery selection was reported to double in another group receiving consultation-based education $(24,46)$.

In some studies, it was noted that maternal intention for natural delivery reduced in control groups receiving routine care in health centers. This finding highlights the inefficacy of routine prenatal care programs in promoting natural childbirth.

Although the aforementioned studies were indicative of increased intention for natural delivery after educational interventions, rate of natural birth was ultimately lower in proportion to the number of women who preferred this delivery mode. In the present review, it was observed that selection of delivery modes is affected by various factors in addition to the knowledge, attitudes and intentions of pregnant women; such examples are physician's opinion, cultural and socioeconomic factors, and abstract norms. According to some of the reviewed articles, physicians had the most significant effect on the selection of childbirth modes by pregnant women $(9,33,35,39,41)$. 
Some of the limitations of the reviewed articles included insufficient explanation of methodologies, inconsistency in the results (texts and tables), ignoring confounders, deficient data analysis, and incomplete results.

The current study was the first narrative review conducted on this subject. One of the limitations of our review was lack of quality assessment of the selected articles and analysis of their results.

\section{Conclusion}

Several studies have denoted the effectiveness of various educational and consultation-based interventions on improving the knowledge, attitudes, and intentions of pregnant women regarding the modes of delivery. However, rate of unnecessary caesarean section remains relatively high in many regions. This could be due to the inconsistent practice of current findings and applied interventions in this regard. On the other hand, effects of other factors (e.g., subjective norms) on the intention and request of pregnant women must be investigated through accurate, high-quality interventions.

Findings of this review could help healthcare authorities with proper decision-making, increasing the quality of health services, and planning for the training of healthcare providers through sufficient educational and consultation programs during pregnancy.

Comparison and combination of various intervention methods require special attention. Furthermore, it is recommended that further high-quality studies, particularly in terms of methodology and data analysis, be conducted in this regard. Recognition of the most effectual educational interventions is possible through performing relevant time series and systematic reviews.

\section{Conflicts of interest}

The authors declare that they have no conflicts interests.

\section{Authors' contributions}

N. Bagherian-Afrakoti contributed in data collec- tion and writing the first draft, M.A. Shirvani involved in revising of content and approving the final manuscript. Also, M. Pourasghar and A. Alipour reviesed the final draft.

\section{Acknowledgments}

Hereby, we extend our gratitude to the Research Deputy and Student Research Committee of Mazandaran University of Medical Sciences.

\section{References}

1. Khawaja M, Choueiry N, Jurdi R. Hospital-based caesarean section in the Arab region: an overview. East Mediterr Health J 2009; 15(2):458-69.

2. Lee AS, Kirkman M. Disciplinary discourses: rates of cesarean section explained by medicine, midwifery, and feminism. Health Care for Women Int 2008; 29(5):448-67.

3. Menacker F, Hamilton BE. Recent trends in cesarean delivery in the United States. New York, US: Department of Health and Human Services, Centers for Disease Control and Prevention, National Center for Health Statistics; 2010.

4. Khani S, Shaabankhani KB. Can the cesarean rate be reduced in Mazandaran? J Mazandaran Univ Med Sci 2005; 15(45):43-50.

5. Malakouti J, Alidoost N, Mohaddesi H, Sattarzadeh Jahdi N, Asghari Jafarabadi M, Salehi Pourmehr H. The evaluating effect of education on knowledge and attitude of nulliparous women toward the delivery method. J Urmia Nurs Midwifery Facul 2014; 12(7):575-68 (Persian).

6. Valiani M, Haghighatdana Z, Ehsanpour S. Comparison of childbirth training workshop effects on knowledge, attitude, and delivery method between mothers and couples groups referring to Isfahan health centers in Iran. Iran J Nurs Midwifery Res 2014; 19(6):653.

7. Moayed Mohseni S, Mohamadi KH. The predictive of cesarean section trend base on present statistic document. Daneshvar 2006; 66(14):59-62 (Persian).

8. Janbabaee G, Moosazadeh M, Agah R, Khani S, Nezammahalleh A, Fallah M. Trend of cesarean section and natural childbirth in governmental and private hospitals during 2007-2014 and its 2021 forecast in Mazandaran province, Iran. J Mazandaran Univ Med Sci 2016; 26(134):1-11 (Persian).

9. Torkzahrani S. Commentary: childbirth education in Iran. J Perinat Educ 2008; 17(3):51-4.

10. Baradaran A. Effect of a participatory intervention to reduce the number of unnecessary cesarean sections performed in Shahrekord of Iran. J Med Sci 2006; 6(4):690-2.

11. Penna L, Arulkumaran S. Cesarean section for non-medical reasons. Int J Gynecol Obstet 2003; 82(3):399-409.

12. Mohammaditabar S, Rahnama P, Mohammadi K. Cesarean 
section on maternal request in Tehran 2010-2011: incidence and predisposing factors. J Mazandaran Univ Med Sci 2014; 24(114):74-82 (Persian).

13. Alimohamadian M, Shariat M, Mahmoudi M, Ramazanzadeh F. The influence of maternal request on the elective cesarean section rate in maternity hospitals in Tehran, Iran. Payesh 2003; 2(2):133-39 (Persian).

14. Garmaroudi GR, Eftekhar H, Batebi A. Factors that affect cesarean among pregnant women. Payesh 2002; 1(2):47-9 (Persian).

15. Rahimikian F, Mirmohamadaliei M, Mehran A, Aboozari $\mathrm{K}$, Barough S. Effect of education designed based on health belief model on choosing delivery mode. Hayat 2008; 14(4):25-32 (Persian).

16. Althabe F, Sosa C, Belizán JM, Gibbons L, Jacquerioz F, Bergel E. Cesarean section rates and maternal and neonatal mortality in low-, medium-, and high-income countries: an ecological study. Birth 2006; 33(4):270-7.

17. Deneux-Tharaux C, Carmona E, Bouvier-Colle MH, Bréart G. Postpartum maternal mortality and cesarean delivery. Obstet Gynecol 2006; 108(3 Pt 1):541-8.

18. Manthata A, Hall DR, Steyn PS, Grove D. The attitudes of two groups of South African women towards mode of delivery. Int J Gynecol Obstet 2006; 92(1):87-91.

19. Koken G, Cosar E, Sahin FK, Tolga Arioz D, Duman Z, Aral I. Attitudes towards mode of delivery and cesarean on demand in Turkey. Int J Gynecol Obstet 2007; 99(3):233-5.

20. Shahraki Sanavi F, Navidian A, Rakhshani F, AnsariMoghadam A. The effect of education on base the theory of planned behavior toward normal delivery in pregnant women with intention elective cesarean. J Med Hormozgan 2014; 18(6):531-9.

21. Vakilian K, Jamilian M. The effect of group training on pregnant women's tendency about the method of delivery and pethidine consumption. Nurs Practic Today 2014; 1(3):135-9.

22. Arjmandi B, Farzinmoqadam S. Study of knowledge pregnant women toward vaginal delivery and cesarean in Tehran. Iran Univ Med Sci J 2007; 14(55):1.

23. Rahnama P, Mohammadi K. Behavioral beliefs about cesarean section according to the theory of planned behavior in pregnant women. J Mazandaran Univ Med Sci 2015; 24(122):169-78 (Persian).

24. Amini F, Parvin Jamshidian Qalehshahi P. The effectiveness of group cognitive behavioral training in changing nulliparous women's attitudes and choice of delivery route. Modern Care J 2015; 12(1):54-60.

25. Khunpradit S, Tavender E, Lumbiganon P, Laopaiboon M, Wasiak J, Gruen RL. Non-clinical interventions for reducing unnecessary caesarean section. Cochrane Database Syst Rev 2011; 15(6):CD005528.

26. Hartmann KE, Andrews JC, Jerome RN, Lewis RM, Likis FE, McKoy JN, et al. Strategies to reduce cesarean birth in low-risk women. Health Care Res Q 2012; 12(13):128.

27. Gagnon AJ, Sandall J. Individual or group antenatal education for childbirth or parenthood, or both. Cochrane Database Syst Rev 2007; 3:CD002869.

28. Green BN, Johnson CD, Adams A. Writing narrative literature reviews for peer-reviewed journals: secrets of the trade. J Chirop Med 2006; 5(3):101-17.

29. Who should use PRISMA? Preferred Reporting Items for Systematic Reviews and Meta-Analyses (PRISMA) website. Available at: URL: www.prisma-statement.org; 2016.

30. Abedian Z, Navaee M, Jaafari SH, Arani A, Ebrahimzadeh S. Comparing the effect of two teaching methods, role playing and lecture on primigravida women's knowledge, attitude and performance according to delivery mode. Iran J Obstet Gyneocol Infertil 2012; 15(1):25-35 (Persian).

31. Lashgari MH, Delavari S, Markazi MN, Gorouhi F. Effects of training programs of pregnant women on their delivery type selection: a single blind, randomized control trial. Ann Military Health Sci Res 2005; 3(4):679-84.

32. Toughyani R, Ramezani MA, Izadi M, Motie Z. The effect of prenatal care group education on pregnant mothers' knowledge, attitude and practice. Iran J Med Educ 2008; 7(2):317-24.

33. Rezakhani Moghaddam H, Shojaeizadeh D, Taghdisi MH, Hamidzadeh Arbabi Y, Savadpour MT. The effect of education by community health volunteers on choice of delivery kind in pregnant women based on the Behavioral Intention Model (BIM). J Sch Public Health Instit Public Health Res 2013; 10(3):27-40.

34. Asfia A, Azam K, Heydarabadi AB, Gilasi HR, Ghanei Z, Dariyani A, et al. The effect of health education on knowledge and attitudes about cesarean in Tehrani women. J Shahid Beheshti Sch Nurs Midwifery 2013; 22(79):16-22 (Persian).

35. Tofighi Niaki M, Behmanesh F, Mashmuli F, Azimi H. The effect of prenatal group education on knowledge, attitude and selection of delivery type in primiparous women. Iran J Med Educ 2010; 10(2):124-30.

36. Sanavi FS, Ansari-Moghaddam A, Shovey MF, Rakhshani F. Effective education to decrease elective caesarean section. J Pak Med Assoc 2014; 64(5):500-5.

37. Arefi Z, Hekamatpou D, Orouji MA, Shaahmadi Z, Khushemehri G, Shaahmadi F. The effect of educational intervention based on BASNEF model on decreasing the cesarean section rate among pregnant women in Khomain Country. J Family Reprod Health 2015; 9(3):101-5.

38. Safari Moradabadi A, Hassani L, Ghanbarnejad A, Madani A, Rajaei M, Dadipoor S. The effect of education on knowledge and preferred method of delivery in nulliparous women. J Health Care 2014; 16(1):74-83 (Persian).

39. Sharifirad GR, Baghiani Moghadam MH, Fathyian Z, Rezaeian M. The effect of health education using behavior intention model on of cesarean in Khomainy-shahr, Iran. Iran J Nurs Midwifery Res 2009; 14(3):105-10.

40. Ghaffari M, Sharifirad G, Akbari Z, Khorsandi M, Hassanzadeh A. Health belief model-based education \& reduction of cesarean among pregnant women: an interventional study. Health Sys Res 2011; 2(7):200-8 (Persian). 
41. Fathian Z, Sharifirad GR, Hasanzadeh A, Fathian Z. Study of the effects of behavioral intention model education on reducing the cesarean rate among pregnant women of Khomeiny-Shahr, Isfahan, in 2006. Zahedan J Res Med Sci 2007; 2(9):124-31 (Persian).

42. Besharati F, Hazavehei SM, Moeini B, Moghimbeigi A. Effect of educational interventions based on theory of planned behavior (TPB) in selecting delivery mode among pregnant women referred to Rasht health centers. Sci J Zanjan Univ Med Sci 2011; 19(77):94-106 (Persian).

43. Kashfi SM, Heydarabadi AB, Hafez AA, Rezaianzadeh A, Shahidi F. Effect of behavioral intention model-based education on decrease the rate of caesarean delivery among pregnant women. J Paramed Sci 2013; 5(1):16-23.

44. Khan-Jeihooni A, Shahidi F, Kashfi SM. Effectiveness of educational program based on the theory of reasoned action to decrease the rate of cesarean delivery among pregnant women in Fasa, Southern Iran. J Educ Commun Health 2014; 1(1):62-71.

45. Baghianimoghadam MH, Hashemifard T, Jafari S, Yadollahi P. The effect of educational intervention on selection of delivery method based on health belief model. J Commun Health Res 2014; 3(2):115-23.

46. Abedi $Z$. The effect of cognitive behavioral group training on the decision of cesarean, to normal delivery of referrers to social security treatment centers in Tehran. J Soc Issu Hum 2015; 3(9):2345-633.

47. Shahnazi H, Sabooteh S, Sharifirad G, Mirkarimi K, Hassanzadeh A. The impact of education intervention on the health belief model constructs regarding anxiety of nulliparous pregnant women. J Educ Health Promot 2015; 4(27):27.

48. Kulkarni A, Wright E, Kingdom J. Web-based education and attitude to delivery by caesarean section in nulliparous women. J Obstet Gynaecol Can 2014; 36(9):768-75.

49. Lagrew DC Jr, Morgan MA. Decreasing the cesarean section rate in a private hospital: success without mandated clinical changes. Am J Obstet Gynecol 1996; 174(1 Pt 1):184-91.

50. Baldwin KA. Comparison of selected outcomes of Centering Pregnancy versus traditional prenatal care. J Midwifery Womens Health 2006; 51(4):266-72.

51. Naeimi N, Gholami M, Qasemi A. A study of awareness and attitudes of pregnant women admitted to social security hospital in Zahedan toward cesarean section. Int $\mathrm{J}$ Anal Pharm Biomed Sci 2015; 4(4):72-80.

52. Young K, Miller YD. Keeping it natural: does persuasive magazine content have an effect on young women's intentions for birth? Women Health 2015; 55(4):447-66.

53. Navaee M, Abedian Z. Effect of role play education on primiparous women's fear of natural delivery and their decision on the mode of delivery. Iran J Nurs Midwifery Res 2015; 20(1):40-6.

54. Rosenstock IM, Strecher VJ, Becker MH. Social learning theory and the health belief model. Health Educ Q 1988; 15(2):175-83.

55. Ajzen I. The theory of planned behavior. Organ Behav Hum Decis Proc 1991; 50(2):179-211.

56. Glanz K, Rimer BK, Viswanath K. Health behavior and health education: theory, research, and practice. New Jersey: John Wiley \& Sons; 2008.

57. Khoursandi M, Vakilian K, Torabi GM, Abdi M. Childbirth preparation using behavioral-cognitive skill in childbirth outcomes of primiparous women. J Babol Univ Med Sci 2013; 15(4):76-80 (Persian).

58. Rajabi GR, Karjo-Kasmaie S, Jabbari H. The effectiveness of cognitive couple therapy on decreasing depression level, and increasing marital satisfaction. J Clin Psycol 2011; 2(4):9-17. 